\title{
Transformer Tuned VCO for MMW Application
}

\author{
Sunanda Ambulker \\ Department of Electronics and Communication \\ Maulana Azad National Institute of Technology \\ Bhopal, India
}

\author{
Sangeeta Nakate \\ Department of Electronics and Communication \\ Maulana Azad National Institute of Technology \\ Bhopal, India
}

\begin{abstract}
Differential cross coupled voltage controlled oscillator with transformer tuning is presented here for millimeter wave (mmw) application. Here Tuning is done by on chip octagonal transformer which is used as inductive element in LC tank. Proposed scheme provides wide Frequency Tuning Range (FTR) up to $7 \mathrm{GHz}$ of bandwidth and gives low phase noise as compared to inductor based oscillator design. In this method transformer tuned VCO is implemented in UMC $65 \mathrm{~nm}$ CMOS Technology which gives FTR from $55 \mathrm{GHz}$ to 62.4 $\mathrm{GHz}$ with phase noise variation -116.5 to $-114.5 \mathrm{dBc} / \mathrm{Hz}$ at 10 $\mathrm{MHz}$ offset.
\end{abstract}

\section{Keywords}

Phase noise, quality factor, Tuning range $\mathrm{VCO}, \mathrm{mmw}$

\section{INTRODUCTION}

In modern wireless communication there is a big requirement to increase the speed of system and Voltage controlled oscillator (VCO) [7] is the most critical and important building block of wireless communication system, in which difficult task is to get mmw frequency with wide tuning range and low Phase Noise (PN). There are so many techniques have been adopted to increase the operating frequency and tuning range of voltage controlled oscillator with low phase noise performance. To gain better phase noise performance LC type of voltage controlled oscillator is being used, but it has narrow tuning range and also mmw frequency generation is a major issue. To get millimeter-wave ( $\mathrm{mmw}$ ) band conventionally, a MOS Varactor in accumulation mode is used in LC type VCOs for frequency tuning [6]. In general to increase the Frequency Tuning Range (FTR) of oscillator multiband operation is normally adopted with reduced VCO gain. To achieve that multiband operation capacitive tuning is being employed which is done by switched capacitor bank, but there are some limitations of capacitive tuning that are produces parasitic capacitance which becomes too large in capacitor bank and also gives low quality factor of capacitor. Now a days new inductive tuning is a substitute of capacitive tuning and it is being used by loaded transformer [1] [9]. Inductive tuning has another advantage that it isolates DC noise from tuning element and have high quality factor.

Rest of the paper is organized as below. Section 2 describes the principle of inductive tuning upon which proposed topology is presented, proposed inductive tuning scheme and EM simulated results are given in section 3.section 4 describes VCO design, simulation results are being presented in section 5, section 6 contains conclusion acknowledgement is provided in section 7 and section 8 gives references.

\section{TRANSFORMER TUNING METHOD}

Principle Behind the inductive tuning [8][9] is explained in Fig 1 Transformer is assumed to be ideal with L1 and L2 as the self inductances of primary and secondary respectively, $\mathrm{k}$ is the coupling coefficient of transformer, Leff is total inductance of the transformer and $\mathrm{Ct}$ is the tuning capacitance which includes Varactor and other parasitic capacitance.

Equivalent circuits Leq and Req can be calculated as

$$
\begin{aligned}
& L_{e q}=L_{1} \frac{R^{2}\left[1-\omega^{2} C L_{2}\left(1-k^{2}\right)\right]^{2}+\omega^{2} L_{2}^{2}\left(1-k^{2}\right)^{2}}{R^{2}\left(1-\omega^{2} C L_{2}\right)\left[1-\omega^{2} C L_{2}\left(1-k^{2}\right)\right]+\omega^{2} L_{2}^{2}\left(1-k^{2}\right)} \\
& R_{e q}=\frac{R^{2} L_{[}\left[1-\omega^{2} C L_{2}\left(1-k^{2}\right)\right]^{2}+\omega^{2} L_{1} L_{2}^{2}\left(1-k^{2}\right)}{R k^{2} L_{2}}
\end{aligned}
$$

Now the Oscillation Frequency is

$$
\omega=\frac{1}{\sqrt{C_{t} L_{e q}}}
$$

\section{PROPOSED TRANSFORMER RESONATOR}

In the proposed type of inductively tuned VCO design Octagonal transformer is used which is implemented in top metal (m9). Single turn transformer is designed for the sake of simplicity with width of $4 \mathrm{um}$ and spacing between primary and secondary being $2.5 \mathrm{um}$. To provide multiple band operation proposed transformer uses MOS switches at primary and secondary of center tapping of the transformer. As shown in fig. 2 equivalent model of transformer with MOS switches at primary and secondary center tapping is given. Simple MOS transistors are used as switches in the circuit..Z1 and $\mathrm{Z} 2$ are the modeled impedances of switches at primary and secondary center tapping respectively. Here the design of transformer is EM simulated in VPCM (Virtuoso passive component designer) which is integrated in Cadence 5.1.41.Fig 3, Fig 4 and Fig.5 gives the layout of octagonal transformer and plot of the transformers primary and secondary inductance for different operating frequencies respectively.

\section{TRANSFORMER TUNED VCO}

The schematic of our transformer tuned VCO design is shown in Fig 6. It consists of two complementary cross coupled differential pairs. Here the performance of this circuit was implemented in a standard $65 \mathrm{~nm}$ CMOS process. This schematic has been chosen because it provides better isolation from the voltage supply and better noise performance.

In the proposed circuit the double cross coupled NMOS (M1, M2) and PMOS (M3, M4) differential pair provides the negative resistance to cancel losses coming from LC tank .MOS transistor in the circuit are biased in such a way that 
resulted transconductance of each of the cross coupled pair is equal that is $-\mathrm{gm} / 2$ and hence total transconductance of the circuit will be -gm. That total transconductance -gm is the negative resistance of the circuit to compensate the loss associated with LC tank. Advantage of use of double cross coupled pair is that less current is used by the circuit that results in lower power consumption. In the proposed circuit design 4 PMOS Varactor is used to tune the different. Frequency band obtained from switching ON and OFF the NMOS switches connected at the center tapping position of the octagonal transformer. By switching ON/OFF switches we get three bands of frequency for mmw application. Main function of the switches in this circuit is that while switching ON/OFF a switch, current will flow in that coil which then induced in secondary coil so the effect is that total inductance (Leq) and resistance (Req) will get change according to the equations presented in section 2 depending on the positions of switches.

\section{SIMULATION RESULTS}

Proposed circuit is simulated in cadence virtuoso 5.1.41 and EM simulation of transformer is carried out in VPCM (Virtuoso Passive Component Designer).The circuit generate stable periodic signal. The results obtained from the simulation of the Transformer based LC tank VCO design show that the phase noise have been drastically reduced. Fig 7 shows the variation of phase noise at frequency of 62.33 for the offset of $1 \mathrm{KHz}$ to $10 \mathrm{MHz}$ and fig 8 give plot for FTR. To compare the performance of previously published oscillators, and FOM (Figure of merit) we have used method by Ham and Hajimiri [8], It is defined by equation (4)[2]:

$$
\text { FOM }(\text { FigureofMerit })=P N-20 \log \left(\frac{f_{0}}{\Delta f}\right)+10 \log \left(\frac{P_{\text {diss }}}{1 m W}\right)
$$

Where $\Delta f$ is the offset frequency, $f_{0}$ is the oscillating frequency, $\mathrm{PN}$ is the phase noise at the offset frequency and Pdiss is the power dissipation of the VCO.

Table I summarizes VCO performance and comparison with other wideband VCO

\section{CONCLUSION}

In this work a transformer based LC tank VCO is designed and analyzed for mmw application with the advantage of wide FTR and low phase noise from variation -116.5 to -114.5 $\mathrm{dBc} / \mathrm{Hz}$ at $10 \mathrm{MHz}$ offset for the tuning range of $55 \mathrm{GHz}$ to $62.4 \mathrm{GHz}$.Figure of merit (FOM) obtained is from -182.8239 $\mathrm{dBc} / \mathrm{Hz}$ to $-181.4564 \mathrm{dBc} / \mathrm{Hz}$. Here at $1.1 \mathrm{~V}$ power supply average power is $13 \mathrm{nW}$.

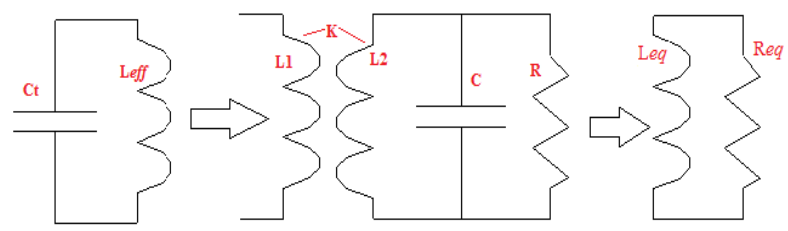

Figure 1 Equivalent circuit model for inductive tuning principle

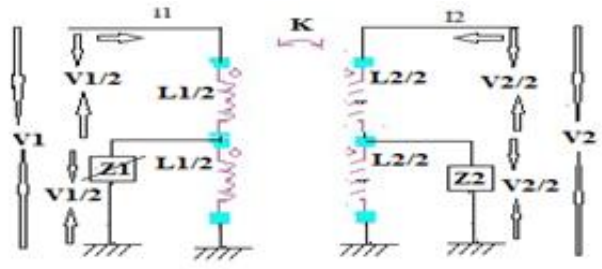

Figure 2 Proposed Transformer Mode

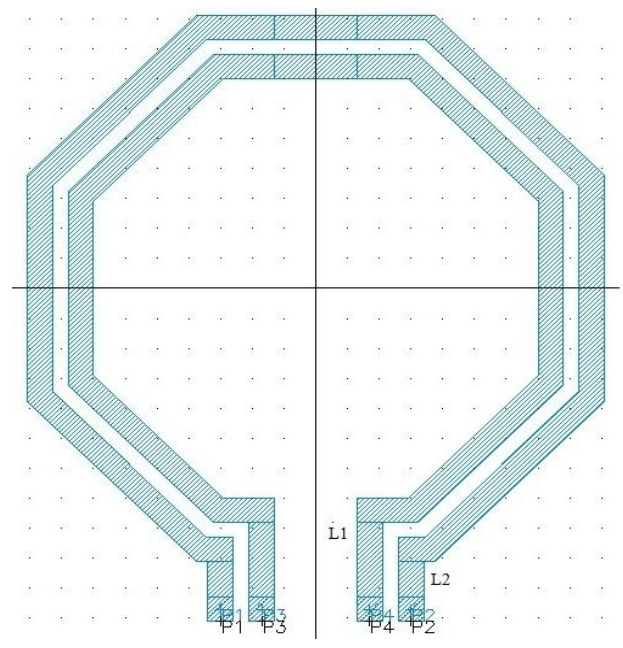

Figure 3 Layout of Octagonal Transformer

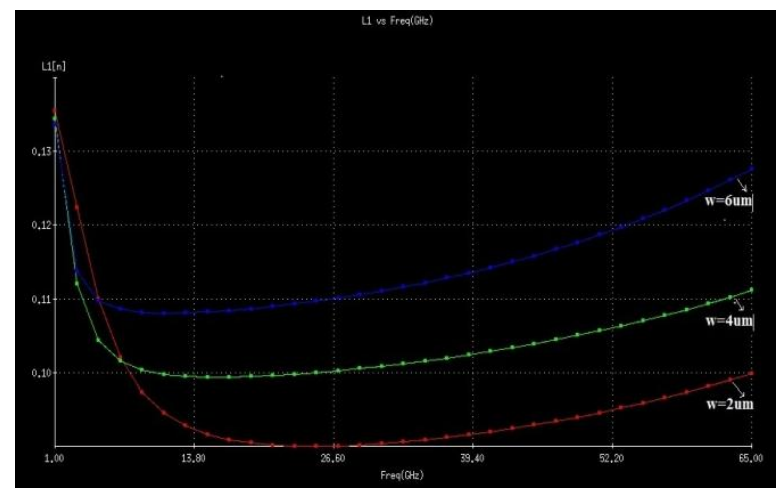

Figure 4 L1

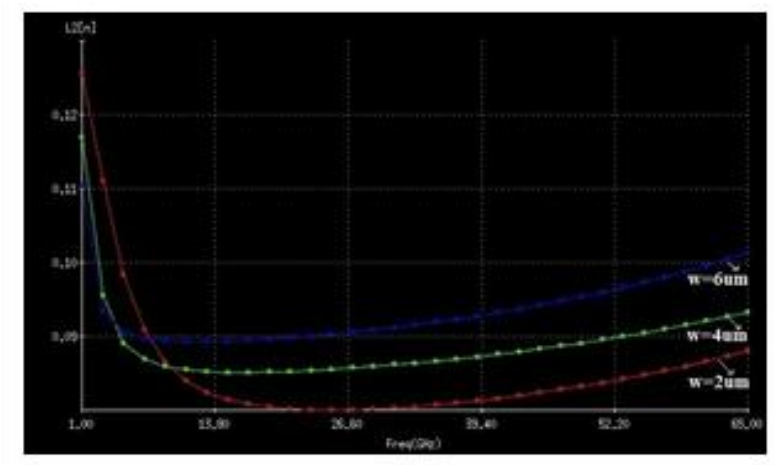

Figure 5 L2 


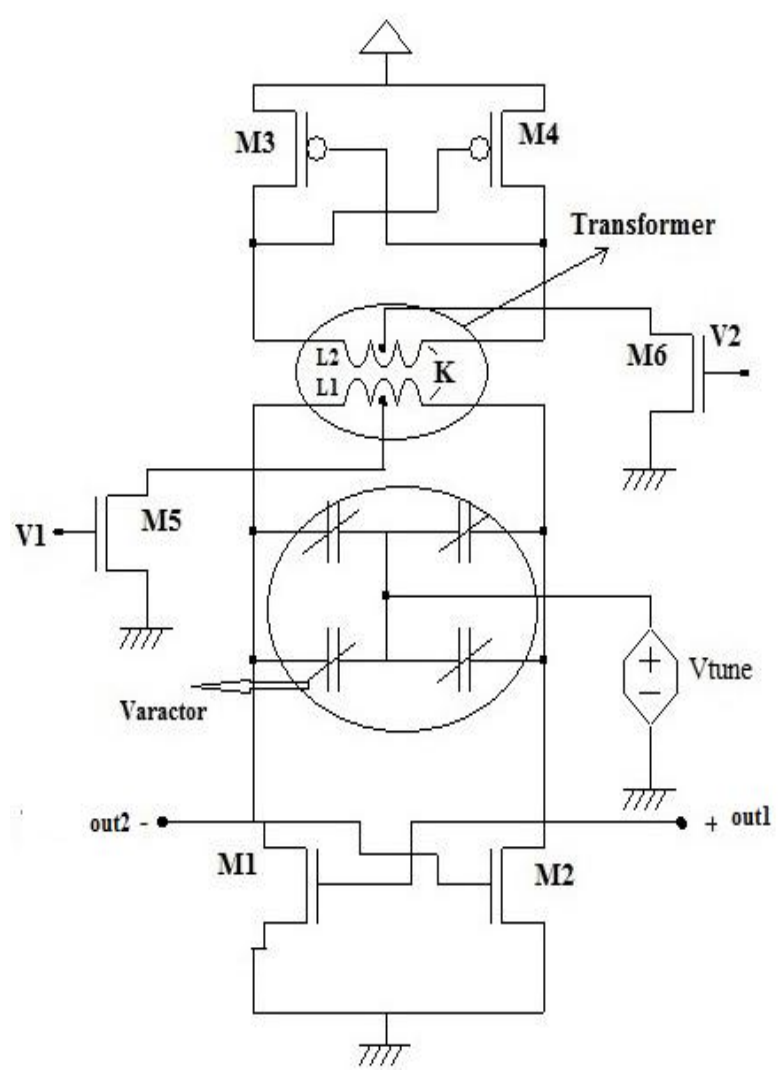

Figure 6 Transformer tuned VCO

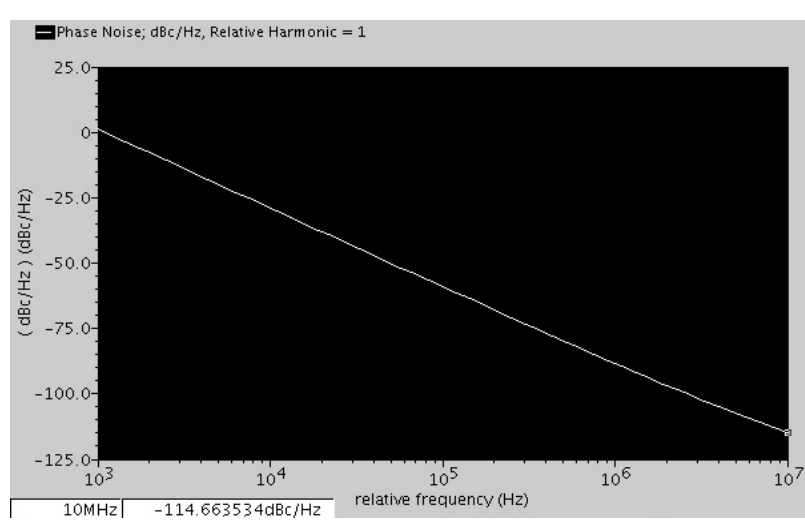

Figure 7 measured phase noise of transformer tuned VCO at $62.3 \mathrm{GHz}$

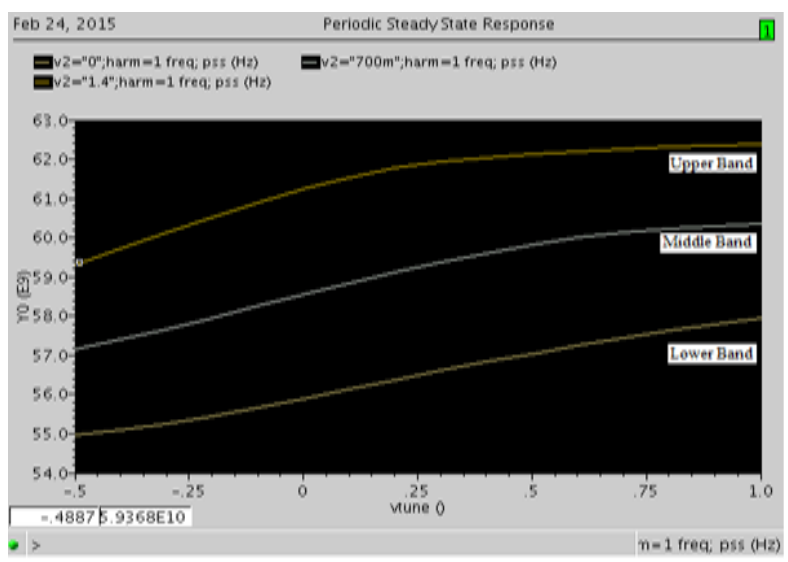

Figure 8 Tuning range of proposed VCO with change in control voltage from- $0.5 \mathrm{v}$ to $1 \mathrm{v}$

Table I Performance summary and Comparison

\begin{tabular}{|c|c|c|c|c|c|c|c|c|}
\hline Ref. & Tech. & $\begin{array}{c}\text { Centre } \\
\text { Freq.(GHz) } \\
\end{array}$ & $\begin{array}{c}\text { TR.(Tuning } \\
\text { range) }\end{array}$ & $\begin{array}{l}\text { PN Ref } \\
\text { Freq. }\end{array}$ & $\begin{array}{c}\text { PN@10MHz } \\
\text { (dBc/Hz) }\end{array}$ & $\begin{array}{r}\text { Power } \\
(\mathbf{m w})\end{array}$ & $\begin{array}{c}\text { FOM } \\
(\mathrm{dBc} / \mathrm{Hz})\end{array}$ & $\mathbf{V}_{\mathrm{DD}}(\mathbf{V})$ \\
\hline [3] & $\begin{array}{l}90 \mathrm{~nm} \\
\text { CMOS }\end{array}$ & 61.7 & $4.9 \%$ & 61.7 & -110 & 1.2 & -185 & 1.2 \\
\hline [4] & $130 \mathrm{~nm}$ & 62.1 & $10 \%$ & 62.1 & -115 & 3.9 & -185 & 1.0 \\
\hline [5] & $130 \mathrm{~nm}$ & 69.7 & $11.19 \%$ & 66 & -107 & 32 & -163 & NA \\
\hline This work & $65 \mathrm{~nm}$ & 58.7 & $12.6 \%$ & 62.3 & -114.66 & 13 & -189.1 & 1.1 \\
\hline
\end{tabular}

\section{ACKNOWLEDGMENTS}

The author would like to thank the VLSI Lab under SMDP project run by MANIT for providing facility to implement the proposed VCO and thanks to all my coordinating faculties for their support and guidance.

\section{REFERENCES}

[1] R. Duarte and J. Fernandes, "A Comparative Study on Transformer and Inductor Based LC Tanks for VCOs", International Journal of Microelectronics and Computer Science Vol.1, No.1, July 2010, pp. 37-41.

[2] D.Leeson, "A simple model of feedback oscillator noise spectrum" in Proc. IEEE, vol. 54, Feb. 1966, pp. 329330.
[3] L. Li et al., "Design and analysis of a $90 \mathrm{~nm}$ mm-wave oscillator using inductive-division LC tank," IEEE J. Solid-State Circuits, vol. 44, no.7, Jul. 2009, pp. 19501958.

[4] U. Decanis et al., "A low-noise quadrature VCO based on magnetically Coupled resonators and a wideband frequency divider at millimeter waves," IEEE J. SolidState Circuits, vol. 46, no. 12, Dec. 2011, pp. 2943-2954.

[5] Z. Liu, E. Skafidas, and R. Evans, "A $70 \mathrm{GHz}$ VCO with $8 \mathrm{GHz}$ Tuning Range in 0.13_m CMOS Technology," Proc. The 3rd European Microwave Integrated Circuits Conf., Oct. 2008. 
[6] K. Ishibashi, M. Motoyoshi, N. Kobayashi, and M. Fujishima, "76 GHz CMOS voltage-controlled oscillator with 7\% frequency tuning range," in Symp. VLSI Circuit Dig. Tech. Papers, Jun. 2007, pp. 176-177.

[7] D. Ham and A. Hajimiri, "Concept and methods in optimization of integrated LC VCOs," IEEE Journal of Solid-State Circuits, vol. 36, no. 6, pp. 896-909, June 2001.

[8] Wei Fei, Hao Yu,Haipeng Fu,Junyan Ren and Kiat Seng Ye "Design and Analysis of Wide Frequency-Tuning
Range $60 \mathrm{GHz}$ VCO By Switching Inductor Loaded Transformer", IEEE Transactions on Circuits and Systems-I: Reg. Paper, vol. 61,no 3,March 2014 ,pp.699711.

[9] S. Rong and H. C. Luong, "Analysis and design of transformer-based dual-band VCO for software-defined radios," IEEE Trans. Circuits Syst. I, Reg. Papers, vol. 59, no. 3, Mar. 2012, pp. 449-462. 